\title{
Status epilepticus due to cerebral air embolism after the Valsalva maneuver
}

Hyun Ji Lyou, MD; Hye Jeong Lee, MD; Grace Yoojin Lee, MD; Won-Joo Kim, MD, PhD

Department of Neurology, Gangnam Severance Hospital, Yonsei University College of Medicine, Seoul, Republic of Korea

\section{CASE REPORT \\ Journal of Neurocritical Care \\ Received: March 11, 2019 \\ Revised: May 3, 2019 \\ Accepted: May 27, 2019 \\ Corresponding Author: \\ Won-Joo Kim, MD \\ Department of Neurology, Gangnam \\ Severance Hospital, Yonsei University \\ College of Medicine, 211 Eonju-ro, \\ Gangnam-gu, Seoul 06273, Republic \\ of Korea \\ Tel: +82-2-2019-3324 \\ Fax: +82-2-3462-5904 \\ E-mail:kzoo@yuhs.ac}

Background: Cerebral air embolism is uncommon but potentially causes catastrophic events such as cardiac damage or even death. However, due to a low overall incidence, it may go undiagnosed.

Case Report: A 56-year-old man with a medical history of right upper lobectomy due to lung cancer showed changes in mental status after the Valsalva maneuver, followed by status epilepticus during admission. Brain and chest computed tomography showed cerebral air embolism and accidental pneumothorax in the right major fissure. After antiepileptic drug infusion and oxygen therapy, he recovered completely.

Conclusion: Since cerebral air embolism may result in fatal outcomes, it should be suspected in patients with sudden neurological deterioration after routine medical procedures.

Keywords: Status epilepticus; Embolism, air; Pneumothorax; Valsalva maneuver

\section{INTRODUCTION}

Cerebral air embolism is a rare but potentially severe complication of iatrogenic procedures or destructive lung disease, possibly resulting in neurological disorders such as encephalopathy, stroke, or seizure. Due to a low overall incidence, cerebral air embolism may go undiagnosed. We report the case of a patient with cerebral air embolism presenting with status epilepticus after the Valsalva maneuver during a pulmonary function test.

\section{CASE REPORT}

A 56-year-old man with a medical history of right upper lobectomy due to lung cancer presented to the emergency room with changes in mental status after the Valsalva maneuver during a pulmonary function test. He had undergone lobectomy 10 days before the event. The oxygen saturation was $99 \%$ by full face mask, and the patient underwent endotracheal intubation because of comatose mental state.

Brain computed tomography (CT) after 30 minutes from the symptom onset revealed scattered air bubbles in the bilateral cere- 
bral subarachnoid space and deep cerebral white matter, suggesting air embolism (Fig. 1A). In addition, chest CT revealed localized pneumothorax in the right major fissure (Fig. 2).

With the intravenous administration of fluid and applied $\mathrm{O} 2$ therapy for 8 hours, the patient was able to make eye contact. However, about 11 hours after the event, three attacks of generalized tonic-clonic seizures with right gaze deviation occurred for $40 \mathrm{~min}$ - utes. The patient did not regain consciousness between the seizures. Suspecting status epilepticus with acute kidney injury, antiepileptic drug with levetiracetam 1,500 mg intravenous was loaded, followed by postictal confusion over 8 hours. Within the 8 hours, he showed tactile and visual extinction, left-sided weakness, and bilaterally positive Babinski signs.

Five days after the seizure, brain magnetic resonance imaging re-
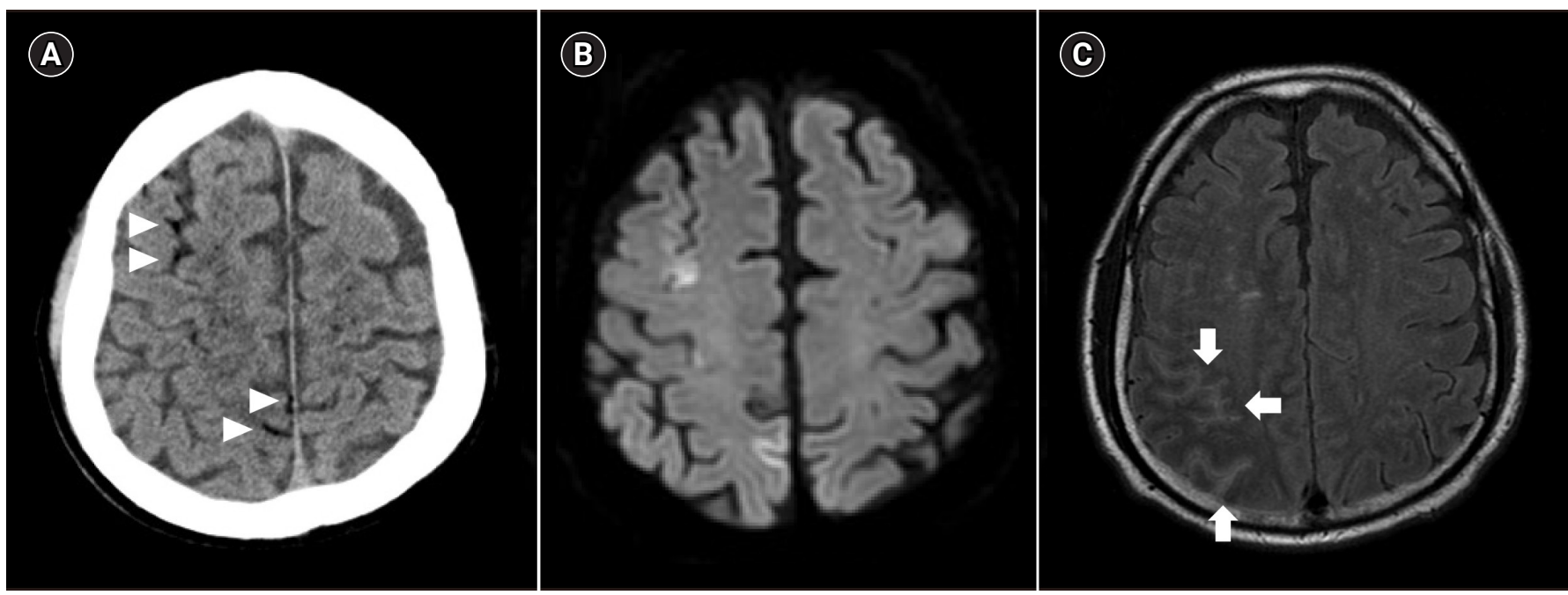

Fig. 1. (A) Brain computed tomography 30 minutes after symptom onset; multiple air bubbles observed prominently in the right parenchymal and subarachnoid vessels (arrowheads). (B, C) Brain magnetic resonance imaging after 5 days after symptom onset; diffuse leptomeningeal enhancement of the right frontal, bilateral parietooccipital, and bilateral cerebellum with diffuse enhancement in the perivascular space of deep white matter (arrows).
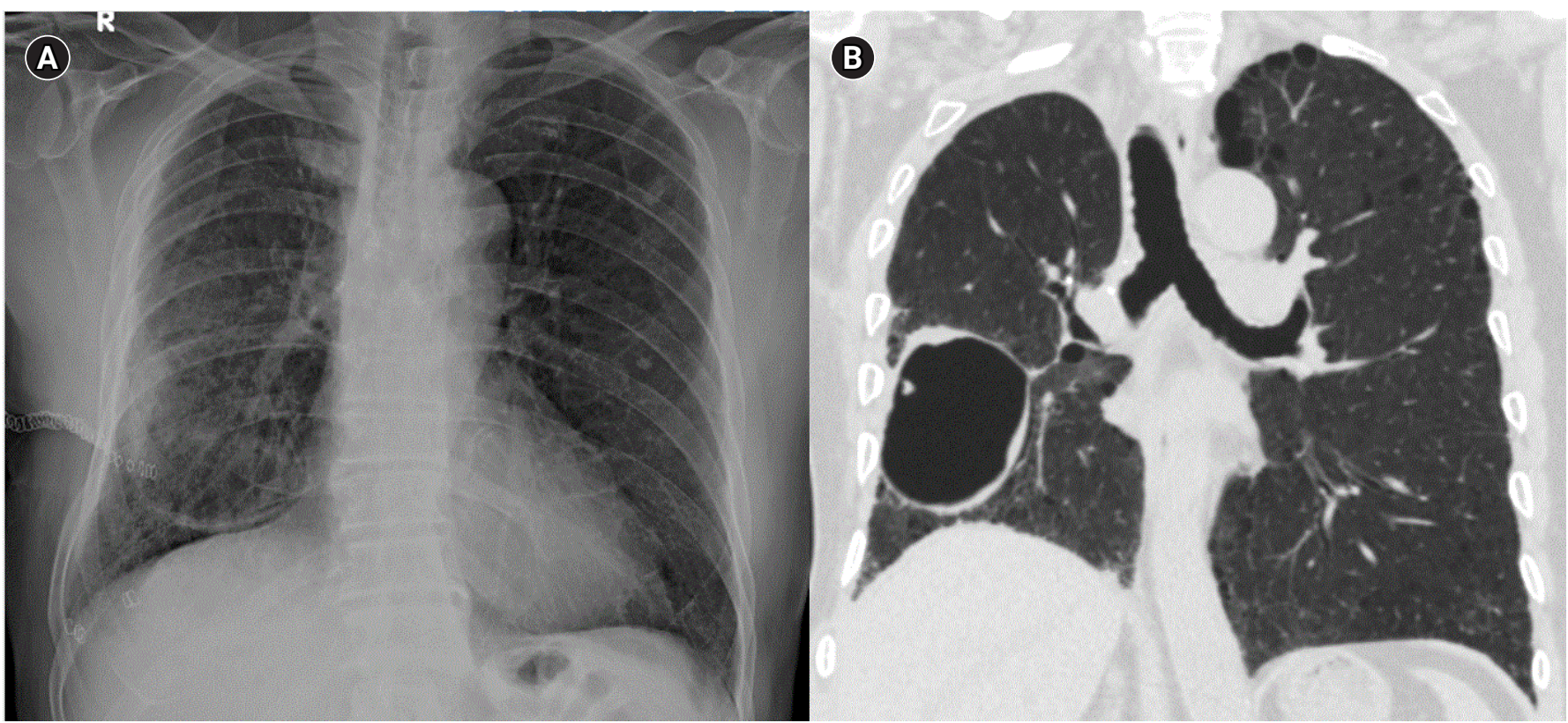

Fig. 2. (A, B) Chest computed tomography; localized pneumothorax in the right major fissure. 
vealed newly appearing diffuse leptomeningeal enhancement of the right frontal, bilateral parietooccipital, and bilateral cerebellum with diffuse enhancement in the perivascular space of bilateral deep white matter (Fig. 1B, 1C). Magnetic resonance angiography revealed mild atherosclerotic changes in the bilateral proximal and cavernous internal carotid arteries and focal stenosis at left M1. Furthermore, a transesophageal echocardiogram revealed patent foramen ovale (PFO) Grade I. Five days after supportive therapies such as mechanical ventilation and oxygen therapy, the patient was discharged from the hospital without any neurological deficits. After discharge, he took levetiracetam $500 \mathrm{mg}$ twice a day daily.

\section{DISCUSSION}

Cerebral air embolism is a rare but life-threatening event, which may cause sudden-onset respiratory distress or neurological events such as seizure and comatose mental status [1,2]. It often occurs with lung trauma or during vascular interventions or surgical procedures such as central venous catheter removal, cardiac surgeries, and endoscopic operations $[3,4]$.

Diagnosis of cerebral air embolism should be based on the patient's medical history and clinical suspicion. Brain CT may be a valuable tool if the air is visible within the cerebral parenchyma or subarachnoid space early in the disease course. However, there is no established imaging technique for the definite diagnosis of cerebral air embolism [5].

In our case, status epilepticus was caused by acute cerebral damage after cerebral air embolism. Anticonvulsant medication may be required for the control of seizures. Otherwise, prophylactic use of lidocaine not only controls seizures but also reduces infarct size and prevents cardiac arrhythmias associated with air embolism [6].

There are two mechanisms behind cerebral air embolism. One is the retrograde venous cerebral embolism via the venous system [1]. In our case, during the Valsalva maneuver, the increased intrathoracic pressure and tension pneumothorax may have provided a pressure gradient for the air to enter the pulmonary veins, resulting in air embolus. The other is the paradoxical embolism in the presence of right-to-left shunting such as $\mathrm{PFO}$ and pulmonary arteriovenous fistula $[5,7-10]$. The conditions that elevate the pulmonary artery pressure enable the air bubbles to move from right to left. The presence of intracardiac shunt (PFO G1) in this patient may explain the cause of air embolism.

The initial treatment consists of immediate volume expansion, administration of $100 \%$ oxygen by face mask, and hyperbaric oxygen therapy $[1,5]$. Hyperbaric oxygen therapy is the adjunct therapy for cerebral air embolism by removing the volume of air embolus and improving the tissue oxygenation. The side effects of hy- perbaric oxygen therapy are rare but can occur by oxygen toxicity on the cardiovascular and neurological systems. Hyperoxic convulsions are exceptionally rare and due to the reperfusion phenomenon.

Iatrogenic gas embolism is a serious disease, with a crude mortality rate of $25 / 119$ (21\%) at 1 year [11]. Therefore, cerebral air embolism should be treated as soon as possible, and immediate therapy has been reported to decrease the mortality rate to $7 \%$ [12].

To the best of our knowledge, this is the first case of status epilepticus caused by cerebral air embolism after the Valsalva maneuver. In addition, this report emphasizes the need for awareness of suspected cerebral air embolism following routine medical procedures to avoid poor prognosis.

\section{ARTICLE INFORMATION}

\section{Conflict of interest}

No potential conflict of interest relevant to this article.

\section{ORCID}

Hyun Ji Lyou, https://orcid.org/0000-0001-9020-8558

Won-Joo Kim, https://orcid.org/0000-0002-5850-010X

\section{Author contributions}

Conceptualization: HJL and WJK. Data curation \& Formal analysis: HJL, HJL, GYL, and WJK. Visualization \& Writing-original draft: HJL. Writing-review editing: HJL, HJL, GYL, and WJK.

\section{REFERENCES}

1. Bou-Assaly W, Pernicano P, Hoeffner E. Systemic air embolism after transthoracic lung biopsy: a case report and review of literature. World J Radiol 2010;2:193-6.

2. Green BT, Tendler DA. Cerebral air embolism during upper endoscopy: case report and review. Gastrointest Endosc 2005;61:620-3.

3. Ho AM, Ling E. Systemic air embolism after lung trauma. Anesthesiology 1999;90:564-75.

4. Lin C, Barrio GA, Hurwitz LM, Kranz PG. Cerebral air embolism from angioinvasive cavitary aspergillosis. Case Rep Neurol Med 2014;2014:406106.

5. Akhtar N, Jafri W, Mozaffar T. Cerebral artery air embolism following an esophagogastroscopy: a case report. Neurology 2001;56:136-7.

6. Jain KK. Textbook of hyperbaric medicine. 5th ed. Cambridge: Hogrefe; 2009. p. 578.

7. Kim SK, Jun IG, Jang DM, Lim J, Hwang GS, Kim YK. Cerebral 
air embolism and subsequent transient neurologic abnormalities in a liver transplant recipient following the removal of the pulmonary artery catheter from the central venous access device: a case report. Korean J Anesthesiol 2016;69:80-3.

8. Schlimp CJ, Loimer T, Rieger M, Lederer W, Schmidts MB. The potential of venous air embolism ascending retrograde to the brain.J Forensic Sci 2005;50:906-9.

9. van Hulst RA, Klein J, Lachmann B. Gas embolism: pathophysiology and treatment. Clin Physiol Funct Imaging 2003;23:23746.
10. Finsterer J, Stöllberger C, Bastovansky A. Cardiac and cerebral air embolism from endoscopic retrograde cholangio-pancreatography. Eur J Gastroenterol Hepatol 2010;22:1157-62.

11. Bessereau J, Genotelle N, Chabbaut C, Huon A, Tabah A, Aboab J, et al. Long-term outcome of iatrogenic gas embolism. Intensive Care Med 2010;36:1180-7.

12. Shi L, Zhang R, Wang Z, Zhou P. Delayed cerebral air embolism complicating percutaneous needle biopsy of the lung. Am J Med Sci 2013;345:501-3. 